\title{
RELAÇÃO ENTRE DOR E ATIVIDADE ELÉTRICA \\ NA PRESENÇA DE BRUXISMO
}

\section{Relation between pain and electric activity in the presence of bruxism}

\author{
Flávia Leães de Almeida ${ }^{(1)}$, Ana Maria Toniolo da Silva ${ }^{(2)}$, \\ Eliane Castilhos Rodrigues Correa ${ }^{(3)}$, Angela Ruviaro Busanello ${ }^{(4)}$
}

\begin{abstract}
RESUMO
Objetivo: mensurar a atividade elétrica dos músculos masseter e temporal, verificar a graduação de dor à palpação e sua correlação com a atividade elétrica em sujeitos com bruxismo. Método: todos os sujeitos do estudo foram selecionados após avaliação por meio do instrumento Critérios de Diagnóstico em Pesquisa para Desordens Temporomandibulares (RDC/TMD) além de avaliação odontológica e fonoaudiológica. A atividade elétrica dos músculos mastigatórios foi avaliada por meio da eletromiografia de superfície nas situações de repouso, máxima intercuspidação e mastigação habitual ritmada. Para a coleta e análise dos sinais eletromiográficos foram usados os Software Biolnspector 1.8 (Lynx $^{\circledR}$ ), e software AqDAnalysis ${ }^{\circledR} .7 .0$ (Lynx $^{\circledR}$ ), respectivamente, quantificados em RMS (raiz quadrada média) e expressos em $\mu \mathrm{V}$ (microvolts). A análise estatística dos dados foi realizada por meio do Coeficiente de Spearman com significância de $p<0,05$. Resultados: os resultados mostraram que o padrão eletromiográfico dos músculos estudados apresentou-se próximos aos níveis de normalidade, no repouso, máxima intercuspidação e na mastigação habitual ritmada. A maioria dos sujeitos apresentou queixa de algum tipo de dor, sendo mais evidenciado o grau severo e, principalmente, no músculo masseter. Não evidenciou-se correlação estatisticamente significante entre dor e atividade EMG, uma vez que esta última mostrou-se próxima aos níveis de normalidade. Conclusão: a análise dos resultados indicou que, apesar da presença da dor nos músculos mastigatórios, a maioria das correlações entre dor e EMG apresentou-se fraca e sem significância estatística, indicando que a dor não interferiu no desempenho da atividade elétrica dos músculos estudados nas situações avaliadas.
\end{abstract}

DESCRITORES: Bruxismo; Eletromiografia; Músculos Mastigatórios; Dor facial

\section{INTRODUÇÃO}

O bruxismo pode ser descrito como um hábito parafuncional de ranger e/ou apertar os dentes

(1) Fonoaudióloga; Especialista em Fonoaudiologia pelo Conselho de Ensino, Pesquisa e Extensão da Universidade Federal de Santa Maria, RS, Brasil; Mestre em Distúrbios da Comunicação Humana pela Universidade Federal de Santa Maria, Santa Maria, RS, Brasil.

(2) Professora Associada da Universidade Federal de Santa Maria, Santa Maria, RS, Brasil; Doutora em Distúrbios da Comunicação Humana pela Universidade Federal de São Paulo.

(3) Professora Adjunto da Universidade Federal de Santa Maria, Santa Maria, RS, Brasil; Doutora em Biologia BucoDental pela Universidade Estadual de Campinas.

(4) Fonoaudióloga; Mestre em Distúrbios da Comunicação Humana da Universidade Federal de Santa Maria.

Conflito de interesses: inexistente que ocorre durante o dia, ou à noite, consciente ou inconsciente e que, por não ser funcional, pode causar, principalmente, dor muscular e danos aos dentes ${ }^{1-4}$.

Sua etiologia abrange fatores locais, sistêmicos, psicológicos, ocupacionais e/ou fatores hereditários ${ }^{5}$. Quanto à prevalência do bruxismo, até o momento, não existe na literatura ${ }^{6}$ um consenso a este respeito, embora haja referência de que o mesmo atinge de 5 a $20 \%$ da população em alguma fase da vida sendo, freqüentemente, associado a sinais de ansiedade, estresse e transtornos do sono'.

Os sinais e sintomas mais expressivos do bruxismo são sensibilidade ao toque, sensação de rigidez nos músculos mastigatórios, contração excessiva e atípica do masseter, cefaléia que, provavelmente, é provocada pela má circulação no 
músculo temporal, desgaste nas facetas dos dentes anteriores e ruído noturno. Além disso, a musculatura mastigatória é largamente afetada com aumento do tônus, espasmos, contração sustentada e perda dos períodos de repouso muscular ${ }^{7,8}$.

O bruxismo está vinculado à etiologia da dor facial ${ }^{9}$, o que vem ao encontro às principais queixas de sujeitos com bruxismo que são: mialgia do masseter e temporal, cefaléia, cervicalgia e dor ou hipersensibilidade dentária a estímulos térmicos ${ }^{10,11}$.

A fonoaudiologia é uma das ciências que contribui para o tratamento do bruxismo favorecendo, principalmente, o relaxamento da musculatura envolvida e melhorando o padrão mastigatório das pessoas que realizam esse hábito, embora o mesmo seja estudado por diversas outras áreas, não há como frear seus efeitos nocivos, sendo apenas possível minimizá-los ${ }^{12}$. Ainda assim, até o momento não há na fonoaudiologia, ao menos como rotina, a realização de avaliações que considerem todos os aspectos que devem ser investigados nos sujeitos que realizam este hábito.

Com o intuito de contemplar estes aspectos, embora ainda de modo perceptivo a aplicação de métodos como o Critério de Diagnóstico em Pesquisa para Desordens Temporomandibulares $(\mathrm{RDC} / \mathrm{TMD})^{13}$ tem sido um importante recurso auxiliar de diagnóstico, pois permite identificar, em vigília, alguns sinais e sintomas do bruxismo.

Entretanto, como a avaliação clínica baseia-se na análise qualitativa, acredita-se que a inclusão de métodos quantitativos, como a eletromiografia de superfície, na rotina de procedimentos de avaliação, vem a contribuir de forma mais efetiva para o diagnóstico, já que possibilita a análise da atividade elétrica dos músculos envolvidos ${ }^{14}$.

Baseando-se nestas colocações o presente estudo teve como objetivo mensurar a atividade elétrica dos músculos masseter e temporal, verificar a graduação de dor à palpação e sua correlação com a atividade elétrica em sujeitos com bruxismo.

\section{MÉTODO}

Participaram desse estudo somente sujeitos que estiveram de acordo com as explicações prestadas pela pesquisadora sobre os procedimentos a que seriam submetidos e que assinaram voluntariamente o Termo de Consentimento Livre e Esclarecido.

Assim, fizeram parte desta pesquisa, 20 sujeitos com bruxismo, sendo 4 do sexo masculino (20\%) e 16 do sexo feminino (80\%), na faixa etária entre 19 e 51 anos (média de idade de 26,90 anos), e sem distinção de raças, os quais deveriam apresentar queixa de dor facial, ranger e/ou apertar os dentes e desgaste dentário.

Foram excluídos sujeitos que apresentassem doenças que danificam a musculatura esquelética, que possuíssem sinais evidentes de comprometimento neurológico que pudessem interferir na compreensão dos procedimentos, bem como na atividade muscular e, também, sujeitos com falhas dentárias.

Aqueles que preencheram os critérios estabelecidos foram submetidos, em um primeiro momento, a uma avaliação pelo instrumento Critério de Diagnóstico em Pesquisa para Desordens Temporomandibulares $(\mathrm{RDC} / \mathrm{TMD})^{13}$, que é um método padronizado, utilizado como auxiliar no diagnóstico da DTM, o qual foi realizado pela pesquisadora.

A escolha desta avaliação foi baseada no fato de que os sintomas da DTM assemelham-se aos do bruxismo ${ }^{10,11}$, além de que, este é um instrumento de qualidade e confiabilidade que permitiu identificar em vigília alguns sinais e sintomas deste hábito, já que as suas manifestações são mais evidentes durante o sono. Este é constituído por dois eixos, sendo que o Eixo I examinou aspectos físicos e o Eixo II avaliou aspectos comportamentais, psicológicos e psicossociais.

A queixa de dor muscular foi confirmada e avaliada pelo exame físico do RDC (Eixo I), por meio da palpação dos músculos temporal e masseter, onde os sujeitos foram orientados a responder, de maneira perceptiva, a sensação que a palpação provocou, classificando-a em ausente (0), dor leve (1), moderada (2) e severa (3), para cada músculo e lado palpado. Embora a pressão à palpação não tenha sido mensurada, o exame foi realizado com pressão em forte intensidade.

Além de investigar a dor muscular, o RDC ratificou a presença de bruxismo nos sujeitos que responderam de modo positivo as questões 15c (Alguém lhe disse, ou você nota, se você range os seus dentes ou aperta os seus maxilares quando dorme a noite?), 15d (Durante o dia, você range os seus dentes ou aperta os seus maxilares?) e 15 e (Você sente dor ou rigidez nos seus maxilares quando acorda de manhã?) do Eixo II do RDC, que verificaram a ocorrência de sintomas freqüentemente evidenciados por estes sujeitos.

Após a avaliação do RDC, os sujeitos realizaram avaliações odontológica fonoaudiológica, e eletromiográfica.

A avaliação odontológica foi realizada por um cirurgião dentista por meio da análise da arcada para verificação de desgaste nos dentes ${ }^{15} \mathrm{com} o$ propósito de confirmar a presença do bruxismo. Esta constatou que dos 20 sujeitos com bruxismo, 3 não apresentavam facetas de desgaste nos dentes, 
realizando apenas apertamento. A avaliação fonoaudiológica, realizada pela pesquisadora, foi composta de uma breve anamnese e exame do sistema estomatognático (SE) e, também, contribuiu para o diagnóstico do bruxismo.

O exame eletromiográfico foi realizado na situação de repouso, máxima intercuspidação e mastigação habitual ritmada para obter registros da atividade elétrica da parte superficial do músculo masseter e da parte anterior do músculo temporal. Os sujeitos foram treinados e tiveram a pele do rosto higienizada com álcool etílico $70 \%$ onde foram colocados eletrodos duplos de superfície de $\mathrm{Ag} / \mathrm{AgCl}$, da marca Hal Ind. e Com. Estes foram posicionados na região de maior volume e massa do músculo, após solicitação da realização de uma contração máxima pelo sujeito para que houvesse uma captação mais efetiva do sinal ${ }^{16}$.

Para evitar quaisquer interferência/ruído ao sinal eletromiográfico, utilizou-se um eletrodo de referência (terra) sobre a região do osso frontal dos sujeitos; foram desligados todos os aparelhos eletrônicos, fontes de luz e, realizado o revestimento do piso e da mesa no local de realização das coletas $^{17}$.

O equipamento utilizado para a realização do exame eletromiográfico foi o modelo EMG 1200 (Lynx Tecnologia Itda.), composto de 8 canais de entrada, placa conversora A/D de 16 bits e faixa de entrada de $+/-2 \mathrm{~V}$. Utilizou-se o filtro do tipo Butterworth, freqüência de corte passa-alta de $10 \mathrm{~Hz}$ e passa-baixa de $1000 \mathrm{~Hz}$ e freqüência de amostragem de $2 \mathrm{KHz}$. Os sinais eletromiográficos foram coletados por meio do Software Biolnspector 1.8 (Lynx), captados por meio de pré-amplificadores ativos com entrada diferencial (PA1020) da Lynx Eletronics Ltda. O ganho foi de 20 vezes, a impedância de entrada de $10 \mathrm{G} \Omega$ e taxa de rejeição de modo comum de $>100 \mathrm{~dB}$.

Durante as coletas, os sujeitos permaneceram sentados confortavelmente, cabeça orientada de acordo com o Plano Frankfurt. Em cada uma das situações avaliadas, foram realizadas 3 coletas, com o propósito de excluir possíveis induções de resultados, bem como para assegurar a fidedignidade do exame ${ }^{17}$.

A prova de repouso foi realizada com o sujeito sentado com posição habitual de lábios e mandíbula, sem contato entre as arcadas dentárias e permanecendo assim por 10 segundos; durante a prova máxima intercuspidação o sujeito deveria contrair a musculatura mastigatória bilateralmente e simultaneamente, com máxima intercuspidação dentária, mordendo com força um pedaço de papel de parafina dobrado em 5 partes (Parafilm®) permanecendo assim por 5 segundos, sob o estímulo verbal da pesquisadora "aperta, aperta, aperta"; na mastigação habitual ritmada o sujeito deveria mastigar de modo habitual pedaços de pão francês, com dimensões de $2 \times 1 \times 1 \mathrm{~cm}$ e com ritmo definido por metrônomo (40 bpm), durante 10 segundos. A escolha do melhor sinal levou em consideração a configuração do mesmo, ou seja, o que apresentasse menos sinais de ruído, bem como o histograma mais harmônico e coerente com sinal selecionado.

Os sinais eletromiográficos foram quantificados em RMS (raiz quadrada média), expressos em $\mu \mathrm{V}$ (microvolts) e salvos em computador portátil com bateria própria. A análise dos dados foi realizada pelo software AqDAnalysis 7.0 (Lynx) e optou-se pela análise do sinal em RMS sem normalização, pois, embora recomendada por permitir a reprodutibilidade dos registros, o uso da mesma pode vir a suprimir distinções entre os sinais coletados ${ }^{17}$. Além disso, considerando que a colocação dos eletrodos nos músculos estudados fica limitada a uma área restrita, a justificativa para normalização pela possibilidade de se recolocar eletrodos em áreas distintas no mesmo músculo em diferentes avaliações, não se aplicaria a músculos com superfície de tamanho reduzida.

Ressalta-se que este estudo foi aprovado pelo Comitê de Ética em Pesquisa da Universidade Federal de Santa Maria - RS, sob o protocolo de número 0172.0.0243.000-07.

A análise estatística dos dados foi realizada por meio do Coeficiente de Spearman o qual estima a correlação de duas variáveis que não tem distribuição conjunta normal. Este varia entre -1 e 1, quanto mais próximo de -1 ou 1 mais forte é a correlação, ocorrendo o contrário quanto mais próximo do zero. O sinal negativo significa que as variáveis são inversamente proporcionais, isto é, as categorias mais elevadas de uma variável estão associadas a categorias mais baixas da outra variável, e o zero evidencia uma correlação nula. $O$ valor de significância para o teste realizado foi de $5 \%(p<0,05)$.

\section{RESULTADOS}

As médias encontradas na avaliação eletromiográfica das provas de repouso, máxima intercuspidação e mastigação habitual ritmada dos músculos masseter direito e esquerdo, e temporal direito e esquerdo, bem como da palpação dessa musculatura durante o exame clínico do RDC/TMD e a correlação entre essas variáveis serão expostas nas Tabelas 1, 2 e 3. 
Tabela 1 - Distribuição das médias e desvio padrão da atividade eletromiográfica (RMS) dos músculos masseteres direito (MD) e esquerdo (ME) e dos músculos temporais direito (TD) e esquerdo (TE) obtidas do grupo de sujeitos com bruxismo $(n=20)$ nas provas da eletromiografia (EMG) de repouso, máxima intercuspidação e mastigação habitual ritmada

\begin{tabular}{cccc}
\hline \multirow{2}{*}{ Músculos } & Repouso & $\begin{array}{c}\text { Máxima } \\
\text { Intercuspidação }\end{array}$ & $\begin{array}{c}\text { Mastigação Habitual } \\
\text { Ritmada }\end{array}$ \\
\cline { 2 - 4 } & Média (DP) & Média (DP) & Média (DP) \\
\hline MD & $3,92(1,07)$ & $268,63(146,99)$ & $71,04(37,02)$ \\
ME & $3,73(1,17)$ & $228,97(156,10)$ & $58,93(35,76)$ \\
TD & $4,63(1,87)$ & $278,02(149,55)$ & $78,52(34,47)$ \\
TE & $4,40(1,51)$ & $244,44(114,48)$ & $71,32(34,88)$ \\
\hline
\end{tabular}

Tabela 2 - Distribuição dos valores absolutos $(n)$ e relativos (\%) dos sujeitos com bruxismo $(n=20)$ segundo a escala de dor do RDC/TMD relatada nos músculos masseteres direito (MD) e esquerdo (ME) e dos músculos temporais direito (TD) e esquerdo (TE)

\begin{tabular}{ccccccccc}
\hline \multirow{2}{*}{ Músculos } & \multicolumn{2}{c}{ MD } & \multicolumn{2}{c}{ ME } & \multicolumn{3}{c}{ TD } & \multicolumn{2}{c}{ TE } \\
\cline { 2 - 10 } & $\mathbf{n}$ & $\%$ & $\mathbf{n}$ & $\%$ & $\mathbf{n}$ & $\%$ & $\mathbf{n}$ & $\%$ \\
\hline Dor 0 & 2 & 10 & 2 & 10 & 4 & 20 & 5 & 25 \\
Dor 1 & 1 & 5 & 2 & 10 & 4 & 20 & 3 & 15 \\
Dor 2 & 4 & 20 & 6 & 30 & 5 & 25 & 7 & 35 \\
Dor 3 & 13 & 65 & 10 & 50 & 7 & 35 & 5 & 25 \\
\hline
\end{tabular}

Tabela 3 - Correlação entre a atividade elétrica dos músculos masseteres direito (MD) e esquerdo (ME) e temporal direito (TD) e esquerdo (TE) com a escala de dor do exame clínico do RDC/TMD dos sujeitos com bruxismo nas provas da eletromiografia (EMG) de repouso, máxima intercuspidação e mastigação habitual ritmada

\begin{tabular}{ccccc}
\hline $\begin{array}{c}\text { Escala de Dor } \\
\text { RDC/TMD }\end{array}$ & MD & M E & T D & TE \\
\hline Repouso & 0,196 & $-0,124$ & $-0,304$ & $-0,189$ \\
Máxima & $-0,097$ & 0,000 & 0,067 & $-0,109$ \\
$\begin{array}{c}\text { Intercuspidação } \\
\text { Mastigação Habitual } \\
\text { Ritmada }\end{array}$ & $-0,283$ & $-0,267$ & 0,000 & 0,268 \\
\hline
\end{tabular}

*significância pelo Coeficiente de Spearman de $p<0,05$

Na Tabela 1 estão expressas a distribuição das médias e os desvios padrões dos músculos MD e ME, e TD e TE nas provas de repouso, máxima intercuspidação e mastigação habitual ritmada dos 20 sujeitos com bruxismo. Os resultados da Tabela 1 mostram que as médias de atividade elétrica, nos músculos avaliados, apresentaramse dentro dos níveis considerados normais em repouso ${ }^{18}$ (próximos de $5 \mu \mathrm{V}$ ) na contração voluntária máxima e na mastigação habitual ritmada.
A Tabela 2 mostra os valores obtidos durante a palpação muscular no exame clínico do RDC/TMD, de acordo com a escala de dor, sendo considerada (0) ausência de dor, (1) dor leve, (2) moderada e (3) severa para cada músculo e lado palpado. Os resultados expostos na Tabela 2 demonstram que, durante a palpação muscular, $90 \%$ dos sujeitos apresentaram queixa de dor (dor 1, 2 e 3) nos músculos MD e ME; $80 \%$ no TD (dor 1, 2 e 3) e $75 \%$ no TE (dor 1, 2 e 3 ). A dor severa (dor 3 ) foi a mais evidente, principalmente, no músculo masseter 
(65\% dos sujeitos no Direito e $50 \%$ dos sujeitos no Esquerdo).

Observa-se na Tabela 3, a correlação entre a atividade elétrica dos músculos, MD e ME, TD e TE, com os resultados da escala de dor referidos na palpação muscular no exame clínico do RDC/ TMD, nos 20 sujeitos com bruxismo nas provas de repouso, máxima intercuspidação e mastigação habitual ritmada.

Os resultados expostos na Tabela 3 evidenciam correlações fracas, inversa para o MD, nas provas de máxima intercuspidação e mastigação habitual ritmada, ou seja, médias de atividade elétrica baixas estão relacionadas a resultados altos na escala de dor; o mesmo ocorreu para o músculo ME na prova de repouso e na prova de mastigação habitual ritmada; no TE nas provas de máxima intercuspidação e repouso e no TD na apenas na prova de repouso ocorreu relação inversa também, embora nestas as médias de atividade elétrica foram altas e estiveram relacionadas a resultados baixos na escala de dor.

Além desses resultados, verificaram-se correlações positivas, ou seja, médias de atividade elétrica e resultados da escala de dor equivalentes, nas provas de repouso, máxima intercuspidação e mastigação habitual ritmada nos músculos MD, TD e TE, respectivamente. No músculo ME na prova de máxima intercuspidação e para o músculo TD na prova de mastigação habitual ritmada, verificou-se correlação nula.

Não foram encontrados resultados estatisticamente significativos em nenhuma das correlações.

\section{DISCUSSÃO}

Os resultados deste estudo (Tabela 1) mostram que, em repouso, os músculos avaliados realizam mínima atividade elétrica, estando dentro dos níveis considerados normais (em torno de $5 \mu \mathrm{V})^{18}$ e concordando com os resultados de estudo anteriormente realizado ${ }^{19}$. Estes resultados diferem da literatura ${ }^{20}$ a qual relata que indivíduos com comprometimento da musculatura mastigatória realizam contrações isométricas constantes, principalmente, por apresentarem uma personalidade estressada, as quais provocam uma alteração no padrão de atividade elétrica dos músculos. Também vão de encontro aos resultados encontrados em outras pesquisas que observaram um aumento da atividade elétrica dos músculos masseter e temporal no repouso em sujeitos patológicos, antes dos mesmos serem submetidos a diferentes tratamentos ${ }^{21,22}$.

Assim como no repouso, observaram-se padrões de atividade elétrica próximos aos normais na máxima intercuspidação e na mastigação habitual ritmada ${ }^{16}$, discordando das afirmações de outra pesquisa ${ }^{23}$, que sugeriu que a presença do bruxismo pode possibilitar a ocorrência de alterações na mastigação, como a diminuição do padrão normal de trituração dos alimentos ou a ocorrência de hipertrofia dos músculos mastigatórios, fatores estes que podem vir a alterar a atividade elétrica dos músculos. Também estão em desacordo com outro estudo que evidenciou aumento da atividade elétrica nos músculos masseteres na situação de contração voluntária máxima em mulheres com bruxismo, a qual esteve associada, segundo os autores, ao estresse provocado pela rotina do trabalho ${ }^{24}$.

Ressalta-se que os resultados deste estudo, nas situações acima descritas, não eram esperados, já que os sujeitos desta pesquisa apresentavam características semelhantes a dos estudos citados como relato de estresse, realização de apertamento dentário e alterações na mastigação, sendo assim, acredita-se que estes aspectos não foram suficientes para modificar o padrão de atividade elétrica dos sujeitos nas situações avaliadas, indicando que, talvez, fatores individuais adaptativos podem ter auxiliado o desempenho da atividade desta musculatura.

Os resultados desta pesquisa (Tabela 2) demonstram que $90 \%$ dos sujeitos apresentaram queixa de dor nos músculos $\mathrm{MD}$ e $\mathrm{ME}, 80 \%$ no TD e $75 \%$ no TE. A dor severa (dor 3 ) foi a que mais se evidenciou, em especial nos músculos masseteres (65\% dos sujeitos no direito e $50 \%$ dos sujeitos no esquerdo). Estes achados comprovam a presença de dor em níveis elevados em indivíduos com bruxismo estando condizentes com a literatura, pois alguns autores descrevem que os hábitos orais deletérios, em especial os hábitos de mordida, como o bruxismo, favorecem a ocorrência de alterações neuromusculares, devido à demanda funcional atípica realizada pela musculatura envolvida, produzindo dor, fadiga e incoordenação, comprometendo não apenas os músculos, como também a mastigação ${ }^{25}$.

A presença de dor mais intensa no músculo masseter justifica-se, pois, em situações que exijam força, espera-se uma ação predominante deste músculo ${ }^{26}$. Assim como neste estudo, a dor já foi relacionada ao bruxismo, ou ao relato de apertar os dentes em outras pesquisas ${ }^{27-29}$. Ressalta-se ainda que no exame do sistema estomatognático dos sujeitos deste estudo, verificou-se predomínio de mastigação assimétrica sendo que esta foi evidenciada em 14 dos 20 sujeitos estudados. Destes 14 sujeitos, 8 apresentaram mastigação à direita, o que, também pode justificar o maior relato de dor severa no músculo masseter direito nos sujeitos 
deste estudo por estes utilizarem mais este lado da face durante a mastigação.

Ainda com relação às médias da atividade dos músculos avaliados durante a máxima intercuspidação, os sujeitos deste estudo apresentaram atividade elétrica com valores próximos aos descritos pela literatura, tanto para sujeitos normais, quanto para sujeitos com alteração $0^{16,30}$, demonstrando que a dor não influenciou no desempenho destes músculos.

Estudo relata que a presença deste hábito pode provocar espasmos, má circulação sanguínea e contrações atípicas nos músculos ${ }^{7}$, aspectos que provocam hipersensibilidade na região em que os mesmos se localizam, podendo provocar uma alteração no padrão de atividade elétrica por dificultar a função dos mesmos, o que não foi evidenciado no presente estudo. Estes resultados também discordam de estudo ${ }^{31}$ que evidenciou redução na atividade elétrica durante a isometria em sujeitos com diagnóstico de dor miofacial antes da realização de um programa de exercício com goma de mascar, além disso, os autores também verificaram decréscimo da dor durante o repouso e mastigação após os exercícios.

As correlações entre dor e atividade elétrica dos músculos avaliados (Tabela 3 ) foram fracas e sem significância estatística. Estes resultados também não são esperados, pois existe na literatura pesquisa ${ }^{27}$ que evidencia esta relação, porém, os resultados encontrados neste estudo podem ser justificados pelo fato das médias de atividade elétrica destes sujeitos estarem próximas aos níveis de normalidade (Tabela 1).

Algumas variáveis como a idade, o sexo, o tempo de descoberta do bruxismo, particularidades de cada sujeito em resistir à dor ou conviver com os efeitos provenientes da presença do hábito, podem mascarar ou acentuar os efeitos da dor sobre a atividade elétrica da musculatura, já que as características individuais de cada sujeito são aspectos relevantes para este tipo de associação.
Em contrapartida este resultado concorda com a literatura ${ }^{32}$ a qual cita que os efeitos desencadeados pelos hábitos como o bruxismo dependerão da natureza, intensidade e duração com que ocorrerem. Embora a dor seja um sintoma físico comumente relatado por sujeitos com bruxismo, ainda que não de maneira geral, já que em alguns estudos essa relação não foi evidenciada ${ }^{33}$, a presença desta não foi um fator agravante para os sujeitos deste estudo, pois não alterou os padrões de atividade elétrica dos músculos avaliados, concordando com estudo que também não evidenciou relação entre mudança na atividade elétrica dos músculos mastigatórios com a dor ${ }^{34}$.

Ressalta-se aqui a importância de trabalhos como este, com uma amostra mais representativa, que verifiquem o desempenho da atividade elétrica dos músculos da mastigação frente a relatos de dor, já que esta relação ainda não está bem estabelecida. Dessa forma pode-se contribuir para diagnóstico mais preciso bem como de alerta para integridade da função da mastigação e estruturas associadas.

\section{CONCLUSÃO}

- O padrão eletromiográfico dos músculos estudados apresentou-se próximos aos níveis de normalidade, em sujeitos com bruxismo no repouso, máxima intercuspidação e na mastigação habitual ritmada.

- A queixa de dor nos sujeitos com bruxismo foi evidente, sendo a dor severa a mais relatada, principalmente no músculo masseter.

- A análise dos resultados indicou que, apesar da presença da dor nos músculos mastigatórios, as correlações entre dor e atividade elétrica mostrou-se fraca e sem significância estatística.

Os resultados indicam que a dor não interferiu no desempenho da atividade elétrica dos músculos estudados nas situações avaliadas. 


\section{ABSTRACT}

Purpose: to measure the electric activity of the masseter and temporal muscles, to verify the graduation of the pain to palpation and its correlation with the electric activity in subjects with bruxism. Method: all subjects were examined according to the Research Diagnostic Criteria for Temporomandibular Disorders (RDC/TMD) instrument combined with the dentistry and the speech pathology evaluation. The electric activity in the masticatory muscles was measured based on surface electromyography in the following situations: during rest, maximum intercuspation and during usual mastication rhythmic. In the collection and analysis of the electromyography signals, it was used Biolnspector ${ }^{\circledR}, 1.8\left(\right.$ Lynx $\left.^{\circledR}\right)$, and AqDAnalysis ${ }^{\circledR}, 7.0\left(\right.$ Lynx $\left.^{\circledR}\right)$, and the data were quantified on RMS (root mean square) and expressed in $\mu \mathrm{V}$ (microvolts). The statistic analysis was based on the Spearman Coefficient with $5 \%$ significance level. Results: the results showed that EMG pattern of the studied muscles was found next to the levels to normality in the rest, maximum intercuspidation and in the rhythmic habitual mastication. Most of the subjects with bruxism had a complaint of some pain, being more evidenced the severe degree and mainly in the masseter muscle. There was not evidenced statistically significant correlation between pain and activity EMG, once that this last one was next to the normality levels. Conclusion: the analysis of the results indicated that in spite of the presence of pain in the masticatory muscles, most of the correlations between pain and EMG were weak and without statistical significance, indicating that pain did not intervene with the performance of the electric activity of the studied muscles in the evaluated situations.

KEYWORDS: Bruxism; Electromyography; Masticatory Muscles; Facial Pain

\section{REFERÊNCIAS}

1. Lavigne GJ, Khoury S, Abe S, Yamaguchi T, Raphael K. Bruxism physiology and pathology: an overview for clinicians. J Oral Rehabil. 2008;35(7):476-94.

2. Branco RS, Branco CS, Tesch RS, Rapoport A. Freqüência de relatos de parafunções nos subgrupos diagnósticos de DTM de acordo com os critérios diagnósticos para pesquisa em disfunções temporomandibulares (RDC/TMD). R Dental Press Ortodon Ortop Facial. 2008; 13(2):61-9.

3. Rodrigues CK, Ditterich RG, Shintcovsk RL, Tanaka O. Bruxismo: uma revisão da literatura. Publ. UEPG Ci. Biol. Saúde. 2006; 12(3): 13-21.

4. Merighi LBM, Silva MMA, Ferreira AT, Genaro KF, Berretin-Felix G. Ocorrência de disfunção temporomandibular (DTM) e sua relação com hábitos orais deletérios em crianças do município de Monte Negro - RO. Rev. CEFAC. 2007; 9(4): 497-503.

5. Carvalho SCA, Carvalho ALA, Lucena SC, Coelho JPS, AraújoTPB. Associação entre bruxismo e estresse em policiais militares. Rev. odonto ciênc. 2008; 23(2): 125-9.

6. Macedo CR. Bruxismo do Sono. R Dental Press Ortodon Ortop Facial. 2008;13(2):18-22.

7. Jardini SRS. A adequação dos músculos orofaciais com o uso dos Exercitadores Pró-Fono. Barueri (SP): Pró-Fono; 2007.
8. Cesar GM, Tosato JP, Gonzalez TO, BiasottoGonzalez DA. Postura cervical e classes oclusais em bruxistas e indivíduos assintomáticos de $\mathrm{dtm}$. Revista de Odontologia da Universidade Cidade de São Paulo. 2006; 18(2)155-60.

9. Bretan O, Nogueira EA. Distúrbios Temporomandibulares e Alterações da Musclatura Mastigatória. Arq. Int. Otorrinolaringol. 2005;9(2):318.

10. Nagamatsu-Sakaguchi C, Minakuchi H, Clark GT, Kuboki T. Relationship between the frequency of sleep bruxism and the prevalence of signs and symptoms of temporomandibular disorders in an adolescent population. Int $\mathrm{J}$ Prosthodont. 2008;21(4):292-8.

11. Svensson $P$, Jadidi $F$, Arima $T$, Baad-Hansen $L$, Sessle BJ. Relationships between craniofacial pain and bruxism. J Oral Rehabil. 2008;35(7):524-47.

12. Coelho JPS, Lucena SC, Carvalho ALA, Lopes FF , Oliveira AEF. Bruxismo do sono e sua associação com distúrbios do sono em policiais. Cienc. Odontol. Bras. 2009; 12 (1): 31-6.

13. Dworkin SF, Leresche L. Research diagnostic criteria for temporomandibular disorders: review, criteria, examinations and specifications, critique. J Craniomandib Disord. 1992;6(4):301-55.

14. Jardini SRS, Ruiz LSR, Moysés MAA. Electromyographic analysis of the masseter and buccinator muscles with the Pro-Fono Facial Exerciser use in bruxers. J. Crânio. 2006;24(1):23-9. 
15. Junqueira $P$. Avaliação e diagnóstico fonoaudiológico em motricidade oral. In: Ferreira LP, BefiLopes DM, Limongi SCO. Tratado de Fonoaudiologia. São Paulo (SP): Roca; 2004.

16. Ferrario VF, Sforza C, Miani JRA, D'addona A, Barbini E. Electromyographic activity of human masticatory muscles in normal young people. Statistical evaluation of reference values for clinical applications. Journal of Oral Rehabilitation. 1993;20(3):271-80.

17. De Luca CJ. The use of surface electromyography in biomechamics. Journal of applied biomechanics. 1997;13(2):135-63.

18. Cram J R, Kasman GS, Holtz J. Introduction to Surface Electromyography. Maryland: Aspen Publishers; 1998.

19. Oncins MC, Freire RMAC, Marchesan IQ. Mastigação: análise pela eletromiografia e eletrognatografia. Seu uso na clínica fonoaudiológica. Distúrbios da Comunicação. 2006;18(2):155-65.

20. Turcio KHL, Garcia AR, Derogis AR, Zuim PRJ. Avaliação eletromiográfica e eletrovibratográfica antes e após o tratamento da desordem temporomandibular. PGRO-Pós-Grad Rev Odontol. 2002;5(2):36-43.

21. Dallanora LJ, Faltin PP, Inoue RT, Santos VMA, Tanaka J. Avaliação do uso de acupuntura no tratamento de pacientes com bruxismo. RGO. 2004;52(5):333-9.

22. Shinozaki EB, Paiva G, Zanin FAA, Junior AB. Avaliação eletromiográfica de pacientes com DTM após a laserterapia. RGO. 2006;54(4):334-9.

23. Soares ISQ, Miranda AFV, Assencio-Ferreira VJ, Di Ninno CQMS. Bruxismo: desempenho da mastigação em adultos jovens. Rev CEFAC. 2004;6(4):358-62.

24. Ferreira LMA, Amorim CF, Giannasi,LC, Nacif SR, Oliveira CS, Silva AM et al. Electromyographic analisys of masseter women presenting sleep bruxism after occlusal splints wearing: a pilot study. Fisioterapia em Movimento. 2007;20(3):123-30.

25. Sousa MA. Estudo eletromiográfico comparativo dos músculos masseter, temporal e orbicular da boca entre jovens com maloclusão classe I, 1a divisão e com oclusão normal [Dissertação]. Piracicaba: Universidade Estadual de Campinas. Mestrado em Ortodontia; 2004.

26. Felício CM. Desenvolvimento normal das funções estomatognáticas. In: Ferreira LP, Befi-Lopes DM, Limongi SCO. Tratado de Fonoaudiologia. São Paulo (SP): Roca; 2004.

27. Glaros AG, Burton E. Parafunctional clenching, pain, and effort in temporomandibular disorders. J Behav Med. 2004;27(1):91-100.

28. Rossetti LMN, Pereira de Araújo CR, Rossetti PHO, Conti PCR. Association between rhythmic masticatory muscle activity during sleep and masticatory myofascial pain: a polysomnographic study. J Orofac Pain. 2008a;22(3):190-200.

29. Guarda-Nardini L, Manfredini D, Salamone M, Salmaso L, Tonello S, Ferronato G. Efficacy of botulinum toxin in treating myofascial pain in bruxers: a controlled placebo pilot study. Cranio: The Journal of Craniomandibular Practice. 2008;26(2):126-35.

30. Rodrigues-Bigaton D, Berto R, Oliveira AS, Bérzin F. Does masticatory muscle hyperactivity occur in individuals presenting temporomandibular disorders? Braz J Oral Sci. 2008; 7(24):1497-501.

31. Gavish A, Winocur E, Astandzelov-Nachmias $T$ Gazit E. Effect of controlled masticatory exercise on pain and muscle performance in myofascial pain patients: A pilot study. Cranio: The Journal of Craniomandibular Practice. 2006;24(3):184-90.

32. Silva AMT, Serpa EO, Blanco APF, Höher FP. Ocorrência de hábitos orais viciosos em indivíduos portadores de Deglutição Atípica. Rev. do Centro de Ciências da Saúde. 2002;28(1-2):58-67.

33. Rossetti LM, Rossetti PH, Conti PC, Araujo CR. Association between sleep bruxism and a temporomandibular disorders: a polysomnographic pilot study. Cranio. 2008b;26(1):16-24.

34. Van Selms MK, Lobbezoo F, Visscher CM, Naeije M. Myofascial temporomandibular disorder pain, parafunctions and psychological stress. J Oral Rehabil. 2008;35(1):45-52.

doi: 10.1590/S1516-18462011005000026

RECEBIDO EM: 09/03/2010

ACEITO EM: 13/09/2010

Endereço para correspondência:

Flávia Leães de Almeida

Rua Maurício Cardoso, 110/03

Alegrete - RS

CEP: 97545-000

E-mail: flavinafono@yahoo.com.br 